\title{
Inwieweit war Russlands Anschluss der Krim historisch gerechtfertigt? Zur Problematik „realistischer" Annexionsnarrative
}

https://doi.org/10.1515/sirius-2018-2006

\section{Einleitung}

Im Sommer 2017 sorgte während des Bundestagswahlkampfs eine Aussage des Parteichefs der deutschen Liberalen, Christian Lindner, für Furore. ${ }^{1}$ Der Vorsitzende der Freien Demokratischen Partei (FDP) - bis dahin für ihre Befürwortung der Einhaltung des Völkerrechts und gesamteuropäischer Integration einschließlich der Ukraine bekannt ${ }^{2}$ - meinte, man solle die russische Annexion der Krim als ein „dauerhaftes Provisorium“ akzeptieren. ${ }^{3}$ Dieser Kommentar Lindners war letztlich nichts Besonderes, brachte der FDP-Chef damit doch nur eine Sichtweise zum Ausdruck, die stillschweigend von vielen, ja womöglich den meisten Politikern, Diplomaten und Journalisten Deutschlands und anderer westlicher Länder geteilt wird. Tatsächlich gehört Lindner - zumindest im politischen Kontext Deutschlands - bei weitem nicht zu jenen, die dem Expansionismus des Kremls mit größter Nachsicht begegnen.

So erklärte etwa der ehemalige Ministerpräsident Brandenburgs und kurzzeitige Bundesvorsitzende der SPD, Matthias Platzek, Ende 2014, die Annexion der Krim durch Russland solle „nachträglich völkerrechtlich gere-

1 Richard Herzinger: „Die FDP ist und bleibt eine Umfallerpartei“, Die Welt, 19. September 2017.

2 Vgl. Andreas Umland: Der neue deutsche Außenminister und die europäische Perspektive der Ukraine, Ukraine-Nachrichten, 28. September 2009; https://ukraine-nachrichten.de/andreas-umland-neuedeutsche-au\%C3 \%9Fenminister-europ $\%$ C3 \%A4ische-perspektiveukraine_1791\#G5C6e1VwPdIKKYI2.99.

3 „Dauerhaftes Provisorium“ Krim. Lindner will Moskau entgegenkommen, NTV, 5. August 2014. Abrufbar unter: www.n-tv.de/politik/ Lindner-will-Moskau-entgegenkommen-article19969480.html

*Kontakt: Dr. Andreas Umland, Senior Research Fellow, Institute for Euro-Atlantic Cooperation, Kyiv, email: umland@stanfordalumni.org gelt werden." ${ }^{4}$ Alexander Gauland, Bundessprecher der rechtspopulistischen Alternative für Deutschland (AfD), die im September 2017 in den Bundestag einzog, verlautete in jenem Sommer gar: „Die Krim ist nun einmal ur-russisches Territorium, und sie kann nicht zurück zur Ukraine. “5 Damit machte sich Gauland voll und ganz das irredentistische Narrativ des Kremls zu eigen. Die KoFraktionsvorsitzende der Partei Die Linke im Bundestag, Sarah Wagenknecht, hatte bereits Anfang März 2014 die Bundesregierung aufgefordert, das Ergebnis des „KrimReferendums“, welches die Annexion der Krim legitimieren sollte, zu akzeptieren, noch bevor dieses am 16. März 2014 stattfand. ${ }^{6}$

\section{Expansionstoleranz im europäischen Mainstream}

Der FDP-Vorsitzende war daher mit seinem seinerzeit skandalträchtigen Kommentar noch relativ moderat. Lindner erklärte lediglich, dass im Unterschied zum Streitpunkt Donbas (Donezbecken) die Rückgabe der Krim an die Ukraine ein langfristiges Projekt sei. Sein scheinbar pragmatischer Ansatz war offensichtlich nicht nur eine Reaktion auf die strikte Weigerung Moskaus, eine Rückgabe der Krim an die Ukraine auch nur zu erwägen, und darauf, dass die Annexion von vielen Russen begeistert aufgenommen wurde. Lindners Haltung ist symptomatisch für eine allgemeine Tendenz im politischen Establishment der EU, Nachsicht gegenüber russischem Impe-

4 Ex-SPD-Chef Platzeck will Annexion der Krim anerkennen, Der Spiegel, 28. November 2014. Abrufbar unter: www.spiegel.de/politik/deutschland/ukraine-krise-matthias-platzeck-will-legalisierungkrim-annexion-a-1003646.html

5 AFD-Parteitag. „Die Krim ist nun einmal ur-russisches Territorium“, Die Welt, 17. Juni 2017.

6 Sahra Wagenknecht warnt vor dem „dritten Weltkrieg“, Der Tagesspiegel, 12. März 2014. Abrufbar unter: www.tagesspiegel.de/ politik/krim-krise-sahra-wagenknecht-warnt-vor-dem-dritten-weltkrieg/9605202.html 
rialismus zu üben und dafür notfalls auch die tatsächliche Geschichte sowie heutige Lage der offiziell oder inoffiziell von Russland okkupierten Territorien zu ignorieren.

Wie unter anderem eine jüngere Analyse des European Council on Foreign Relations (ECFR) zeigte, gibt es eine Reihe etablierter EU-Parteien, die regelmäßig dubiose russische Positionen unterstützen oder zumindest tolerieren. Gustav Gressel vom Berliner ECFR-Büro stellte 2017 fest: „Diese Parteien bekennen sich uneingeschränkt zum westlichen Modell, zur offenen Gesellschaft, zu Freihandel, politischen Freiheiten, gesellschaftlicher Modernisierung und einem säkularen Staat. Aber sie treten zugleich für engere Beziehungen oder wirtschaftliche Zusammenarbeit mit Russland ein, sie plädieren bei der ersten Gelegenheit für die Lockerung von Sanktionen, oder sie vertreten zweideutige Standpunkte, wenn es um die Frage geht, wie die europäische Sicherheitsarchitektur gestaltet werden sollte." 7

Die vom Kreml propagierte einhellige Unterstützung unter den Bewohnern der Krim und das Narrativ einer angeblich historisch gerechtfertigten Annexion sind daher nicht nur bei antiamerikanischen Publizisten und Politikern zu finden, sondern auch in den westlichen politischen Mainstream eingedrungen. Beide Argumentationslinien sind besonders populär unter den zahlreichen „Russlandverstehern" in EU-Wirtschaftskreisen, osteuropakundlich unbedarften Salonexperten und bei Vertretern verschiedener populistischer Parteien, ob nun am rechten oder linken Rand. ${ }^{8}$ Sie blenden nicht nur die Tatsache aus, dass Moskaus handstreichartige Besetzung der Halbinsel ab Ende Februar 2014 der formalen Grenzverschiebung vorausging; somit waren nicht die so genannten „Unabhängigkeitserklärungen“ vom 11. und 17. März oder Russlands offizielle Annexion der Krim am 18. März 2014, sondern das vorherige russische, von Putin später ausdrücklich bestätigte militärische Eingreifen auf der Krim der wichtigste Völkerrechtsbruch. ${ }^{9}$ Zudem gibt es Hinweise darauf, dass das am 16. März 2014 von Russland auf der Krim organisierte „Referendum“ massiv gefälscht war und keinesfalls eine überwältigende Mehrheit der Krimbewohner die „Wiedervereinigung“ Russlands mit der Halbinsel unterstützt hat. Auch fallen die angeblich schwerwiegenden historischen Gründe für den russischen Anschluss der Krim bei näherer Betrachtung in sich zusammen.

7 Gressel 2017.

8 Heinemann-Grüder 2015

9 Heintze 2014; Luchterhandt 2014a, 2014b; Marxsen 2014, 2015; Behlert 2015; Bílková 2015; Grant 2015; Singer 2015; Zadorozhnii 2016.

\section{Die zweifelhaften Ergebnisse des „Referendums“}

Interessantererweise stammt ein besonders kritischer früher Kommentar zu dem Schein-Referendum auf der Krim von drei Vertretern des Rates für die Entwicklung der Zivilgesellschaft und Menschenrechte beim Präsidenten der Russischen Föderation, eines Beratungsorgans von Vladimir Putin. ${ }^{10}$ Eines der Mitglieder dieser offiziellen Institution Russlands, Evgenij Bobrov, hatte Mitte April 2014 die Krim privat besucht. In Folge seiner Beobachtungen und Gespräche während dieses inoffiziellen Aufenthalts auf der gerade annektierten Halbinsel sowie auf Grundlage weiterer Nachforschungen berichteten die drei Mitglieder des Menschenrechtsrats, dass nach Einschätzung „praktisch aller befragten Experten und Einwohner" die Beteiligung am Referendum in der ARK nicht bei 83,1 Prozent, wie von den Kreml-gesteuerten Behörden auf der Krim offiziell angegeben, sondern eher zwischen 30 und 50 Prozent gelegen hat. Von den ARK-Bewohnern, die am Referendum teilnahmen, hätten - gemäß der Einschätzung der drei renommierten russischen Menschenrechtler - nicht 96,77 Prozent für die Annexion gestimmt, wie von den Moskau-ergebenen Behörden behauptet, sondern lediglich 50 bis 60 Prozent. ${ }^{11}$

Letztgenannter Prozentsatz liegt nicht weit entfernt vom durchschnittlich Ergebnis diverser Meinungsumfragen auf der Halbinsel vor der Annexion. ${ }^{12}$ Beim Referendum zur ukrainischen Unabhängigkeit im Dezember 1991 war die Unterstützung auf der Halbinsel zwar landesweit die mit Abstand geringste; doch waren auch in der Republik Krim 54,19 \% und Stadt Sevastopol 57,07 \% der Abstimmenden für die Loslösung der Ukraine von Moskau. Die kritische Bewertung der Abstimmung auf der Krim am 16. März 2014 durch die drei Mitglieder des Menschenrechtsrats beim Präsidenten der Russischen Föderation steht auch im Einklang mit anderen Wahlbeteiligungsanalysen, die eine Verfälschung der Abstimmungsergebnisse nahelegen. ${ }^{13}$ Sie wird bestätigt durch die noch niedrige-

10 Eine Liste westlicher relevanter früher Expertenkommentare zum Pseudoreferendum findet sich im Literaturteil von: Podolian 2015, 122-128.

11 Evgenij A. Bobrov: Problemy žytelej Kryma, Blogi členov Soveta, 22. April 2014. Abrufbar unter: http://president-sovet.ru/members/ blogs/bobrov_e_a/problemy-zhiteley-kryma-/.

12 Eine (unvollständige) englischsprachige Übersicht einiger relevanter Umfragen findet sich hier: https://en.wikipedia.org/wiki/ Crimean_status_referendum,_2014\#Polling. Weiter Umfrageergebnisse bei: Podolian 2015.

13 Kireev 2014; Berezovec‘ 2015. 
ren Schätzungen der Wahlbeteiligung seitens des Medžlis (Rat) der Krimtataren, des offiziellen Exekutivorgans der repräsentativen Versammlung (Qurultai) der indigenen muslimischen Bevölkerung der Halbinsel. ${ }^{14}$

Selbst wenn man von einer deutlich höheren Wahlbeteiligung und einem größeren Rückhalt für die Annexion in der Stadt Sevastopol, als Basis der russischen Schwarzmeerflotte, ausgehen kann, ${ }^{15}$ würde dies bedeuten, dass womöglich weniger als ein Drittel der Gesamtbevölkerung der Krim ihre Stimme für einen Anschluss der Halbinsel an Russland abgegeben hat. Dies wäre ein zu geringer Prozentsatz, um eine derart folgenreiche Änderung der Nachkriegsgrenzen in Europa zu rechtfertigen. Der Bericht der Mitglieder des russischen Menschenrechtsrats zitierte zudem politische Experten auf der Krim, nach denen die „Bewohner der Krim nicht so sehr für einen Anschluss an Russland als für, in ihren Worten, ein Ende ,der grassierenden Korruption und räuberischen Gewalt durch die Donezker Statthalter' gestimmt hatte [das heißt durch die Mitglieder des Janukovyč-Clans, die zwischen 2010 und 2013 auf die Krim entsandt wurden].“

Der renommierte britische Ukraineexperte Andrew Wilson bestätigt die Relevanz dieses Aspektes der Krimsezession:

Janukovyč [...] half dabei, die autonomistischen Tendenzen auf der Krim vor 2014 wiederzubeleben, in dem er derart nachdrücklich seine eigenen Leute an die Macht brachte. Alle - einschließlich der lokalen russischen Nationalisten, Krimtataren und Ukrainophilen - lehnten die Herrschaft der so genannten Makedonier ab („Makedonier“ [...] war ein Wortspiel mit der Stadt Makijivka in Janukovyčs Heimatregion Donezk). Gemäß Rustam Temirgaliev, stellvertretender Vorsitzender des Ministerrats der Krim in der ersten Hälfte von 2014, gab es zwei Trends im Winter 2013-2014: der erste, „die Welle, welche die Donezker entfernte, verband sich mit dem zweiten Trend - Wiedervereinigung mit Russland“. Ablehnung der Makedonier formte die lokale Politik mehr als die weitgehend mythische Gefahr eines „ukrainischen Faschismus“ bevor die russische Intervention das Endresultat bestimmte. ${ }^{16}$

14 Siehe eine kurze englischsprachige Zusammenfassung hier: https://en.wikipedia.org/wiki/Crimean_status_referendum,_2014\# Alternative_estimates_of_results.

15 Bobrov, a.a.O (Fn. 12).

16 Wilson 2017, 6.

\section{Warum spätere Umfragen das „Referendum“ nicht nachträglich legitimieren}

In einer der letzten wirklich aussagekräftigen Umfragen, die Mitte Februar 2014 - nur wenige Tage vor Beginn der Krimbesetzung - durchgeführt wurde, hatten 41 Prozent der Befragten in der ARK (d.h. die Krim ausgenommen Sevastopol) den Zusammenschluss von Russland und der Ukraine zu einem Staat unterstützt. ${ }^{17}$ Dieses Resultat korrespondierte in etwa mit Ergebnissen früherer Befragungen zu einem möglichen Anschluss der Halbinsel an Russland. ${ }^{18}$ Die verschiedenen Umfragen, die nach der militärischen und politischen Übernahme der Schwarzmeerhalbinsel durch Russland durchgeführt wurden, belegen dahingegen scheinbar eine überwältigende Unterstützung der Krimbewohner - d.h. von regelmäßig über oder sogar weit über 80 Prozent - für die Annexion. ${ }^{19}$ Doch haben diese vermeintlich eindeutigen Erhebungsergebnisse nach der Annexion nur eine begrenzte Aussagekraft für die Deutung der Ereignisse auf der Krim Anfang 2014. ${ }^{20}$

Zum einen spiegeln offensichtlich die jüngeren Umfrageergebnisse stärker als die älteren die Auswirkungen der wüsten Hetzkampagne gegen die Ukraine in den kremlkontrollierten Medien wider - der einzigen Masseninformationsquelle, die den Bewohnern der Krim seit März 2014 noch zugänglich ist. ${ }^{21}$ Zum anderen ist zu berücksichtigen, dass Abstimmungsentscheidungen nicht nur von politischen Vorlieben, sondern auch von strategischen Überlegungen bestimmt werden. Bei vielen Befragungen lässt sich eine Präferenz zur Beibehaltung des jeweiligen Status quo beobachten. Dieser Effekt hatte auf der Krim bis Anfang 2014 erhebliche „proukrainische“ Folgen und sicherte die - angesichts zweifellos vorhandener prorussischer Emotionen - erstaunlich hohe politische Stabilität auf der Halbinsel bis zum Beginn der Moskauer Annexionsvorbereitung Anfang 2014. ${ }^{22}$ Selbst viele ansonsten eindeutig prorussisch eingestellte Krimbewohner sprachen sich noch 2012/2013 bei Entweder-Oder-Fragen für einen Verbleib der Halbinsel in der Ukraine - und nicht für einen Wechsel zu Russland - aus. ${ }^{23}$

17 KIIS 2014

18 Podolian 2015.

19 Siehe einige Zahlen, Zitate und Quellen dazu hier: https://en.wiki pedia.org/wiki/Crimean_status_referendum,_2014\#Post-referen dum_polls.

20 Sasse 2017.

21 Fedor 2015.

22 Sasse 2014.

23 Knott 2018. 
Auch darf man nicht die erheblichen Risiken unterschätzen, die sich für die Befragten beim Thema der Zugehörigkeit der Krim ergeben. Die Respondenten auf der okkupierten Halbinsel müssen heute erhebliche Entschlusskraft und Risikobereitschaft aufbringen, um gegenüber Fremden ihre potentielle Kritik an der Annexion, Reue über die Sezession oder gar Unterstützung einer Rückkehr der Krim zur Ukraine zum Ausdruck zu bringen.

Nach ihrer Annexion durch Russland ist die Schwarzmeerhalbinsel zu einer jener Regionen Europas geworden, in welcher der Schutz elementarer politischer und Bürgerrechte nur noch eingeschränkt gewährleistet ist. ${ }^{24}$ Die Missbilligung der Annexion wird heute auf der Krim durch die neuen Behörden und kremlkontrollierten Medien in hohem Maße politisch stigmatisiert und könnte im schlimmsten Fall für solche Respondenten ernsthafte Nachwirkungen haben - wenn, zum Beispiel, Umfragegespräche abgehört oder gar fingiert werden. Auch gilt seit 2014 auf der Krim die besonders harsche russische Antiextremismus- und Antiseparatismusgesetzgebung, welche auf Unterdrückung abweichender politischer Meinungen ausgelegt ist. ${ }^{25}$ Moskau und seine Stellvertreter auf der Krim bedrängen politisch Andersdenkende - insbesondere Mitglieder der krimtatarischen Minderheit ${ }^{26}$ - oder auch bloße Sympathisanten ukrainischer Symbole und Kultur auf der Halbinsel allwöchentlich. ${ }^{27}$ Aus diesen und ähnlichen Gründen können eventuelle Folgen einer Kritik der Annexion, eines Bedauerns der Sezession oder gar eines Bekenntnisses zum ukrainischen Staat von den Befragten in Umfragen kaum noch eingeschätzt werden. Die Ergebnisse wahlsoziologischer Forschung auch renommierter westlicher Institute auf der Krim sind daher seit 2014 mit großer Vorsicht zu betrachten.

\section{Der dubiose Ablauf des „Referendums“}

Es gibt weitere Gründe, warum das von Russland abgehaltene Pseudo-Referendum nicht als Rechtfertigung für eine nachsichtige Haltung gegenüber Russland im Hinblick auf die Annexion der Krim dienen kann. Die Vorbereitungen,

24 UNHCR 2017.

25 Putin Signs Law Punishing Separatism with Jail, Sputnik, 29. Dezember 2013, sputniknews.com/russia/20131229186063866-PutinSigns-Law-Punishing-Separatism-with-Jail/.

26 Halbach 2015.

27 KHPG 2016-2018. der Ablauf, die mediale Begleitung und die Formulierung der Fragen des „Referendums“ waren so offensichtlich tendenziös, dass dieses Abstimmungsverfahren als Lehrbuchbeispiel für eine manipulierte Wahl dienen kann. So wurde etwa das Datum des Referendums in kurzer Zeit zweimal geändert, und die Bürger der Krim hatten weder Zeit noch Gelegenheit, öffentlich, kontrovers und frei die Alternativen zu diskutieren, unter denen sie bei der angeblichen Volksabstimmung am 16. März 2014 auswählen konnten. ${ }^{28}$

Vor dem „Referendum“ hatte die OSZE erklärt, weshalb sie keine Beobachtermission zu dieser Abstimmung entsenden würde: „Internationale Erfahrungen [...] haben gezeigt, dass Prozesse, die auf die Korrektur einer Verfassungsordnung abzielen, und Gespräche über eine regionale Autonomie komplex und zeitraubend sind und sich manchmal über Monate oder sogar Jahre erstrecken [...]. Über politische und rechtliche Anpassungen in dieser Hinsicht müsste in einem inklusiven und strukturierten Dialog auf nationaler, regionaler und lokaler Ebene beraten werden." ${ }^{29}$ Diese Bedingungen waren nicht erfüllt, und aus diesem Grund haben es die OSZE und alle anderen relevanten Wahlbeobachter-Organisationen abgelehnt, ihre Vertreter zur Beobachtung zu entsenden. Stattdessen lud der Kreml Vertreter verschiedener ausländischer radikaler Gruppierungen ein und präsentierte diese der russischen Bevölkerung als internationale Wahlbeobachter. ${ }^{30}$

Die Stimmabgabe fand unter erheblichem psychologischem Druck statt, welchen allgegenwärtige reguläre russische Truppen ohne Abzeichen (,grüne Männchen“beziehungsweise „höfliche Menschen“) und teilweise ebenfalls bewaffnete prorussische irreguläre Verbände ausübten. Seltsamerweise fand sich auf dem Abstimmungsbulletin keine Option einer simplen Aufrechterhaltung des geltenden Status quo, das heißt der seit 1998 gültigen Republiksverfassung der ARK. Die Wähler auf der Krim hatten lediglich die Möglichkeit, entweder für den Anschluss an Russland oder für die Wiedereinführung einer älteren Verfassung der ARK von $1992 \mathrm{zu}$ stimmen. Mehr noch: auch

28 Podolian 2015.

29 OSCE Chair says Crimean referendum in its current form is illegal and calls for alternative ways to address the Crimean issue, OSCE, 11. März 2014. Abrufbar unter: https://www.osce.org/cio/116313.

30 Vgl. Shekhovtsov 2015; s. a. Anton Shekhovtsov: „Pro-Russian Extremists Observe the Illegitimate Crimean 'Referendum'”. Böogbeitrag vom 17. März 2014. Abrufbar unter: http://anton-shekhovtsov. blogspot.com/2014/03/pro-russian-extremists-observe.html; s.a. Halya Coynash: „Myth, 'Observers' and Victims of Russia’s Fake Crimean Referendum,” Human Rights in Ukraine, 16. 3. 2016; http:// khpg.org/en/index.php?id=1458089893\&w=referendum. 
diese beiden Optionen waren unklar formuliert, ja in gewisser Hinsicht absurd.

\subsection{Das ambivalente Versprechen einer „Wiedervereinigung“}

Die erste Option versprach den Krimbewohnern eine „Wiedervereinigung“ (vossedinenie) der Krim mit „Russland“. Die Krim hatte allerdings nie zu einem „Russland“ gehört, das von großen Teilen des Territoriums des postsowjetischen ukrainischen Staats, zu dem die Krim seit 1991 gehörte, getrennt gewesen wäre. Ein Großteil des heutigen Festlands der Ukraine war ungefähr genauso lange wie die Krim Bestandteil zunächst des Zarenreichs und dann der Sowjetunion, also jener Staaten, auf die sich das Wort „Russland“ in dem Referendum offenbar bezog. Von 1783 bis 1991 hatte die Krim lediglich zu einem Imperium gehört, das manchmal „Russland“ genannt wird, jedoch nicht dem heute existierenden russischen Nationalstaat entsprach. ${ }^{31}$

Der größere Teil des gesamten Territoriums der heutigen Ukraine - und nicht nur die Krim - gehörte einst zum Zaren- bzw. Sowjetreich, ebenso wie fast das gesamte Gebiet der heutigen Russischen Föderation. Beide postsowjetische Republiken, die Russische Föderation und die unabhängige Ukraine, sind Nachfolgestaaten jenes „Russlands“, auf das sich das Versprechen einer „Wiedervereinigung “ im Pseudoreferendum von 2014 bezog. Die Halbinsel Krim hatte nie zu einem russischen Nationalstaat gehört, der vor 1991 getrennt von der FestlandUkraine existiert hätte.

Die einzige Landverbindung zwischen der Krim und dem Territorium der heutigen Russischen Föderation während der zaristischen und sowjetischen Periode war das südöstliche Territorium der heutigen Ukraine, über welches auch die Eroberung der Krim durch Katharina die Große im 18. Jahrhundert stattfand. Daher konnte die Krim 1991 nicht von „Russland“ abgetrennt und 2014 auch nicht mit der Russischen Föderation ,wiedervereinigt“ werden. Vielmehr trennte sich die gesamte Ukraine einschließlich der geographisch und historisch zu ihr gehörenden Schwarzmeerhalbinsel 1991 von „Russland.“ Der Kreml spielte und spielt in dieser (wie freilich auch in vieler anderer) Hinsicht mit Begriffen und Konzepten. Etliche westliche Rezipienten fielen und fallen auf diese historischen Manipulationen herein.

Zwar ist es richtig, dass die Bevölkerung der Krim seit ihrem Einschluss ins zaristische und später sowjetische

31 Dazu mehr bei: Furman 2011.
Imperium nie von ethnischen Ukrainern dominiert war. Die Mehrheit der Krimeinwohner wurde zunächst von den heute weitgehend pro-ukrainischen Krimtataren und nach deren schrittweiser Vertreibung bzw. Deportation von der Halbinsel - immer stärker von ethnischen Russen gestellt. Andererseits gehörte die Krim jedoch innerhalb des Zarenreichs seit 1802 zum Gouvernement Taurien. Dieser große Regierungsbezirk schloss nicht nur die Schwarzmeerhalbinsel, sondern auch einen Großteil des südöstlichen Festlands der heutigen Ukraine ein, welches mit der Krim durch den Isthmus von Perekop verbunden ist.

Der südostukrainische Festlandteil des zaristischen Schwarzmeerbezirks war territorial sowie demographisch größer als die Krim und hatte in seiner Gesamtheit eine mehrheitlich „kleinrussische“, d.h. ukrainophone Bevölkerung. ${ }^{32}$ Von den bei der Volkszählung von 1897 ca. 1,4 Millionen Einwohnern des gesamten Taurischen Gouvernements - also sowohl der Krim als auch des Festlandteils nördlich der Halbinsel - waren gut 0,4 Millionen Russischsprecher und gut 0,6 Millionen Ukrainischsprecher. ${ }^{33}$ Von den damals ca. 0,55 Millionen Einwohnern der Halbinsel Krim wiederum waren damals 35,5 Prozent Tataren, 33,1 Prozent Russen und 11,8 Prozent Ukrainer. ${ }^{34}$ Im Ergebnis stellt die über 100 Jahre lange taurisch-zaristische Periode der Krimvergangenheit eher eine verwaltungshistorische Verbindung der Halbinsel zum Territorium der heutigen Ukraine sowie einen Bezug zu den pro-ukrainischen Krimtataren als zum Territorium der heutigen Russischen Föderation und zur russischen Nation her. ${ }^{35}$

Die in Annexionsapologien häufig angesprochene anschließende 32-jährige Zugehörigkeit der Krim zur Russischen Sozialistischen Föderativen Sowjetrepublik (RSFSR) zwischen 1922 und 1954 ist der darauffolgenden 37-jährigen Zugehörigkeit der Krim zur Ukrainischen Sozialistischen Sowjetrepublik (UkrSSR) 1954-1991 gegenüberzustellen. In die RSFSR-Periode der Krimgeschichte fällt zudem die größte und besonders grausame Bevölkerungsveränderung auf der Halbinsel - die von Stalin 1944

32 Holovčenko/Doroško 2016, 75.

33 Pervaja vseobščaja perepis‘ naselenija Rossijskoj Imperii 1897 g. Paspredelenie naselenija po rodnomu jazyku, gubernijam i oblastjam, Demoskop Weekly. Abrufbar unter: http://www.demoscope.ru/ weekly/ssp/rus_lan_97.php?reg=71.

34 Die Zahlen und ihre Quellen finden sich hier: https://en.wikipedia. org/wiki/Crimea.

35 „Ukrainische Historiker weisen [zudem] auf eine lange Geschichte früheren Engagements [vor 1783] hin, während der die ukrainischen Kosaken eine intimere Interaktion mit der Halbinsel hatten, als der nördliche Moskowiter Staat.“ Wilson 2017, 5, bezugnehmend auf: Smoliy 2015. Siehe auch: Hromenko 2016. 
angeordnete massenmörderische Deportation der Krimtataren nach Zentralasien, bei der ein großer Teil des muslimischen Krimvolkes umkam. Die Geschichte der Deportationsaktion und langjährigen Verbannung sowie der anschließenden Rückkehr und Wiedereingliederung der Krimtataren in ihr Heimatland als einem Teil der Ukraine, wie auch der Stalinkult im Putinschen Russland, hat die politischen Präferenzen der Krimtataren, die heute circa 12 Prozent der Bevölkerung der Halbinsel stellen, nachhaltig geprägt. ${ }^{36}$ Sie bewirkt heute eine entschiedene Unterstützung der Souveränität und Integrität des postsowjetischen ukrainischen Staates und ein weitgehendes Einverständnis mit einer Rückkehr der Krim in die Ukraine (und weniger die Forderung nach einer Unabhängigkeit der Krim) seitens der meisten Krimtataren und ihrer politischen Organisationen. Die pro-ukrainische Einstellung der Krimtataren und ihre Deportationsgeschichte rückte für die breite europäische Öffentlichkeit durch den Sieg der krimtatarischen Sängerin Jamala für die Ukraine beim Eurovision Song Contest 2016 mit dem Lied „1944“ kurz ins Zentrum der Aufmerksamkeit. ${ }^{37}$

Die Ereignisse der Sowjetperiode sind, ähnlich den Entwicklungen der vorrevolutionären Zeit, nur bedingt als historische Argumente für die russische Annexion von 2014 geeignet. Sie tun letztlich ohnehin wenig zur Sache. Die Sowjetunion war ein totalitärer Staat, in dem die ökonomisch und geographisch motivierte Verschiebung der Krim von der RSFSR zur UkrSSR 1954 eine rein administrative und keine politische Bedeutung hatte. ${ }^{38}$ Derartige verwaltungstechnische Übereignungen von Territorien gab es in der Geschichte der UdSSR viele.

So hatte die Ukrainische Sowjetrepublik etwa bei einer innersowjetischen Grenzverschiebung im Jahr 1925 ein weit größeres Territorium an die Russische und Belarussische Sowjetrepublik verloren, als sie bei der Übernahme der Krim 1954 gewann. ${ }^{39}$ Niemand in der ukrainischen politischen Elite hat jedoch aufgrund dieser Tatsache oder mit Verweis auf vorrevolutionäre Karten, welche eine weit größere „Ukraine“ als den heutigen ukrainischen Staat zeigen, bisher territoriale Ansprüche an Nachbarländer gestellt. Derartige historische Narrative, megalomanische Visionen und irredentistische Pläne sind in der Ukraine, wie in den meisten Ländern, politischen Randströmungen vorbehalten.

36 Magocsi 2014; Hottop-Riecke 2016; s. a. Natalja Belicer: Kryms'ki tatary jak korinnyy narod. Istorija pytannja i suchasni realiï. Kyïv, 2016. Abrufbar unter: http://mip.gov.ua/files/pdf/blicer_pro_krimtat.pdf.

37 Wilson 2017, 15.

38 Jilge 2015.

39 Holovčenko/Doroško 2016, 91.
Aus diesen und anderen Gründen hatte die postsowjetische russische Staatsführung bis 2014 den Platz der Krim in der postsowjetischen Ukraine - ungeachtet vieler Streitigkeiten - zu keinem Zeitpunkt offiziell in Frage gestellt. Tatsächlich hat sie diesen - trotz zahlreicher gegensätzlicher inoffizieller politischer Anspruchserklärungen russischer Politiker und einiger irredentistischer Deklaration des russischen Parlaments seit 1991 - in mehreren Verträgen und Vereinbarungen ausdrücklich bestätigt. ${ }^{40}$ Die beiden wichtigsten derartigen Dokumente waren das trilaterale russisch-ukrainisch-belarussische Abkommen von Belovež von 1991 über die Auflösung der UdSSR mit dem damaligen Präsidenten Boris Jelzin und das bilaterale russisch-ukrainische Abkommen über den Grenzverlauf zwischen beiden Staaten von 2003 unter Präsident Vladimir Putin. Beide Verträge wurden ordnungsgemäß vom russischen beziehungsweise ukrainischen Parlament ratifiziert und von den jeweiligen russischen beziehungsweise ukrainischen Präsidenten unterzeichnet. In der offiziellen Pressemeldung der russischen Präsidialadministration zu Putins Unterzeichnung des Ratifikationsgesetzes für den Grenzvertrag im Jahr 2004 hieß es: „Die Grundlage der Grenzen sind die administrativen Abgrenzungen zwischen der RSFSR und der Ukrainischen SSR zum Zeitpunkt der Auflösung der UdSSR ...“41

Wenn man dennoch ein „historisches Recht“ Moskaus auf die Krim unter Verweis auf deren Einschluss ins zaristische Reich im Jahr 1783 anerkennen würde, dann müsste man auch einen geschichtlich begründeten Anspruch der heutigen Russischen Föderation auf Annexion eines erheblichen Teils des Territoriums der heutigen FestlandUkraine anerkennen. Ein Großteil der heutigen Zentral-, Ost- und Südukraine waren ungefähr ebenso lange von Moskau kolonisiert, wie die Schwarzmeerhalbinsel. Zudem könnte Moskau dann auch Ansprüche auf weitere Territorien außerhalb der heutigen Russischen Föderation erheben - da sie ungefähr genauso lange oder sogar noch länger zu eben jenem „Russland“ gehört hatten, auf das sich das Pseudoreferendum von 2014 bezog.

\section{Kuzio 2007.}

41 President Vladimir Putin signed a law ratifying the Treaty between Russia and Ukraine on the Russian-Ukrainian State Border, President of Russia, 23. April 2004. Abrufbar unter: www.en.kremlin. ru/events/president/news/30820. 


\subsection{Die unklare Alternative zur Annexion im „Referendum“}

Die zweite Wahlmöglichkeit im „Referendum“, die eine Rückkehr zur „Verfassung der Republik Krim von 1992“ versprach, war noch verwirrender formuliert als die erste Annexionsoption. Das Paradoxon der zweiten Frage an die Krimbewohner bestand darin, dass im Jahr 1992 auf der Krim zwei deutlich verschiedene Verfassungstexte verabschiedet worden waren. ${ }^{42}$ Die Wähler wurden 2014 - ob nun absichtlich oder unabsichtlich - im Unklaren darüber gelassen, auf welche der beiden vorhandenen Annexionsalternativen im „Referendum“ sich ihre Entscheidung bezogen hätte. Sie wurden einfach gefragt: „Sind Sie für die Wiederherstellung der Gültigkeit der Verfassung der Republik Krim von 1992 und den Status der Krim als Teil der Ukraine (kak časti Ukrainy)?“

Die Frage lässt offen, ob mit der Verfassung „,von 1992“ das eher konföderative Grundgesetz der Krim vom Mai 1992 oder die wesentlich abgeänderte, eher föderative Verfassungsvariante vom September 1992 gemeint war. In beiden Verfassungstexten wird die Krim klar als Teil der Ukraine definiert. In der Maiverfassung geschieht dies in Artikel 9 durch die Formulierung „vchodit v gosudarstvo Ukraina“ (wörtl.: „geht in den Staat Ukraine ein“). ${ }^{43}$ In der erheblich modifizierten Septemberversion wird dies zusätzlich in Artikel 1 mit den Worten „V sostave Ukrainy“ (wörtl.: „im Bestand der Ukraine“) zum Ausdruck gebracht. ${ }^{44}$

Hätte bei dem „Referendum“ diese zweite Option gewonnen, wäre es scheinbar den Machthabern überlassen gewesen, eine Wahl zwischen den beiden verschiedenen Grundgesetztexten der ARK aus dem Jahr 1992 zu treffen. Es drängt sich der Verdacht auf, dass diese unorthodoxe zweite Option - statt der näherliegenden Wahlmöglichkeit, den bis dahin geltenden Status quo aufrechtzuerhalten absichtlich schwammig formulierte wurde. Womöglich sollte die Unbestimmtheit der Alternative zur Annexion die Unterstützung für die erste und weit eindeutigere Option Anschluss an Russland - erhöhen. Die Wahl, die die Krimbewohner im März 2014 hatten, war in gewisser Hinsicht

42 Vgl. Andreas Umland: Strannosti kremlevskogo „referenduma“ v Krymu, Novoe vremja, 19. September 2016; https://nv.ua/opinion/ umland/strannosti-kremlevskogo-referenduma-v-krymu-223716. html.

43 Konstitucija Respubliki Krym, Verkhovna Rada Ukraïny, 6. Mai 1992. Abrufbar unter: http://zakon5.rada.gov.ua/krym/show/ rb076a002-92.

44 Zakon Respubliki Krym O vnesenii izmenenij i dopolnenij v Konstituciju Respubliki Krym, Verkhovna Rada Ukraïny, 25. September 1992. Abrufbar unter: http://zakon5.rada.gov.ua/krym/show/ rb0155002-92. weniger eine zwischen Russland und der Ukraine als eine Entscheidung zwischen Klarheit und Unklarheit.

Keine dieser Informationen ist außergewöhnlich, geheim oder originell. ${ }^{45}$ Die aufgeführten Tatsachen und eine Reihe anderer aufschlussreicher Aspekte der denkwürdigen Ereignisse vom Februar-März 2014 sind in der Ukraine und unter Osteuropaexperten an Universitäten und Think-Tanks sowie bei Regierungen und zivilgesellschaftlichen Organisationen im Westen bekannt. Dennoch scheinen viele westliche Beobachter, die schnell mit Kommentaren über die Vergangenheit, Annexion und Zukunft der Krim bei der Hand sind, die meisten dieser Tatsachen nicht zu kennen beziehungsweise zu ignorieren. Stattdessen schenken etliche Kommentatoren dem apologetischen Narrativ des Kreml Glauben: Ein völkerrechtlich zugegebenermaßen holprig initiiertes Referendum habe jedenfalls zu einer Grenzverschiebung geführt, die angeblich von der übergroßen Mehrheit der Krimbewohner damals entschieden gewollt wurde und zudem ein vorgebliches historisches Unrecht wieder gut gemacht hat.

\section{Literatur}

Behlert, Benedikt (2015): Die Unabhängigkeit der Krim. Annexion oder Sezession? Bochum: Institut für Friedenssicherung und Humanitäres Völkerrecht an der Ruhr Universität Bochum, IFHV Working Paper, 5 (2), September. Abrufbar unter: www.ifhv.de/ documents/workingpapers/wp5_2.pdf.

Berezovec', Taras (2015): Aneksija: ostriv Krym. Chroniky “hibrydnoï viyny". Kyïv; Brajt Buks.

Bílková, Veronika (2015): The Use of Force by the Russian Federation in Crimea, Zeitschrift für ausländisches öffentliches Recht und Völkerrecht, 75, 27-50.

Doroško, Mykola (2018): Neohološeni viyny Rosiï proty Ukraïny u XX - na počatku XXI st. Pryčyny i naslidky. Kyïv; Nika-Centr.

Fedor, Julie, Hg. (2015): Russian Media and the War in Ukraine, Journal of Soviet and Post-Soviet Politics and Society, 1 (1), $1-300$.

Furman, Dmitrij (2011): Russlands Entwicklungspfad. Vom Imperium zum Nationalstaat, Osteuropa, 61 (10), S. 3-20.

Grant, Thomas D. (2015): Aggression against Ukraine. Territory, Responsibility, and International Law. London; Palgrave.

Gressel, Gustav (2017): Fellow travellers. Russia, anti-Westernism, and Europe's political parties. London: European Council on Foreign Relations (ECFR) 14. Juli; http://www.ecfr.eu/ publications/summary/fellow_travellers_russia_anti_ westernism_and_europes_political_parties_7213.

Halbach, Uwe (2014): Repression nach der Annexion. Russlands Umgang mit den Krimtataren, Osteuropa, 64 (9-10), 179-190.

45 Ein Fülle von englischsprachigen Informationen findet sich etwa in: Transitions Online 2015. 
Heinemann-Grüder, Andreas (2015): Lehren aus dem Ukrainekonflikt. Das Stockholm-Syndrom der Putin-Versteher, Osteuropa, 65 (4), 3-24.

Heintze, Hans-Joachim (2014): Völkerrecht und Sezession - Ist die Annexion der Krim eine zulässige Wiedergutmachung sowjetischen Unrechts? Humanitäres Völkerrecht. Informationsschriften, 27 (3), 129-138

Holovčenko, Volodymyr/Doroško, Mykola (2016): Hibrydna viyna Rosiï proty Ukraïny. Istoryko-polityčne doslidžennja. Kyïv; Nika-Centr.

Hottop-Riecke, Mieste (2016): Die Tataren der Krim zwischen Assimilation und Selbstbehauptung. Der Aufbau des krimtatarischen Bildungswesens nach Deportation und Heimkehr (1990-2005). Stuttgart; Ibidem Verlag.

Hromenko, Serhiy, Hg. (2016): Naš Krym. Nerosiys'ki istoriï ukraïns'koho pivostrova. Kyïv; K.I.S.

Jilge, Wilfried (2015): Geschichtspolitik statt Völkerrecht. Anmerkungen zur historischen Legitimation der Krim-Annexion in Russland. Bonn: Bundeszentrale für politische Bildung, 27. Februar. Abrufbar unter: www.bpb.de/internationales/ europa/russland/analysen/202223/analyse-geschichtspolitikstatt-voelkerrecht-anmerkungen-zur-historischen-legitimationder-krim-annexion-in-russland.

KHPG (2016-2018): Human Rights Abuses in Russian-Occupied Crimea, Human Rights in Ukraine. http://khpg.org/en/index. php?r=2.5.44.

KIIS (2014): How relations between Ukraine and Russia should look like? Public opinion polls' results. Kiev International Institute of Sociology, (4. März 2014). http://www.kiis.com. ua/ ?lang=eng\&cat=reports\&id=236\&page $=1$.

Kireev, Aleksandr (2014): 0 nepravdobodnoj javki po rajonnam i gorodam Kryma, Livejournal, 16. März. Abrufbar unter: https:// kireev.livejournal.com/1005294.html.

Knott, Eleanor (2018): Identity in Crimea before Annexation. A Bottom-up Perspective, in: Pål Kolstø/Helge Blakkisrud, Hrsg.: Russia Before and After Crimea. Nationalism and Identity, 2010-17. Edinburgh, Edinburgh University Press, 282-305.

Kuzio, Taras (2007): Ukraine - Crimea - Russia. Triangle of Conflict. Stuttgart; Ibidem Verlag.

Luchterhandt, Otto (2014a): Die Krim-Krise von 2014. Staats- und völkerrechtliche Aspekte, Osteuropa, 64 (5-6), 61-86.
Luchterhandt, Otto (2014b): Der Anschluss der Krim an Russland aus völkerrechtlicher Sicht, Archiv des Völkerrechts, 52 (2), 137-174.

Magocsi, Paul Robert (2014): This Blessed Land. Crimea and the Crimean Tatars. Toronto; University of Toronto Press.

Marxsen, Christian (2014): The Crimea Crisis. An International Law Perspective, Zeitschrift für ausländisches öffentliches Recht und Völkerrecht, 74, 367-391.

Marxsen, Christian (2015): Territorial Integrity in International Law - Its Concept and Implications for Crimea, Zeitschrift für ausländisches öffentliches Recht und Völkerrecht, 75, 7-26.

Podolian, Olena (2015): The 2014 Referendum in Crimea, East European Quarterly, 43 (1), 111-128.

Sasse, Gwendolyn (2014): The Crimea Question. Identity, Transition, and Conflict. Cambridge, Mass.; Harvard University Press.

Sasse, Gwendolyn (2017): Terra Incognita. The Public Mood in Crimea, ZOiS Report, 3. Abrufbar unter: www.zois-berlin.de/ fileadmin/media/Dateien/ZOiS_Reports/ZOiS_Report_3_2017. pdf.

Shekhovtsov, Anton (2015): Far-Right Election Observation Monitors in the Service of the Kremlin's Foreign Policy, in: Marlene Laruelle, Hrsg.: Eurasianism and the European Far Right. Reshaping the Europe-Russia Relationship. Lanham; Rowman \& Littlefield, 223-243.

Singer, Tassilo (2015): Intervention auf Einladung, in: Evgeniya Bakalova, Tobias Endrich, Khrystyna Shlyakhtovska, Galyna Spodarets, Hrsg.: Ukraine. Krisen. Perspektiven. Interdisziplinäre Betrachtungen eines Landes im Umbruch. Berlin; Wissenschaftlicher Verlag, 235-260.

Smoliy, Valeriy, Hg. (2015): Istorija Krymu v zapytannjach ta vidpovidjach. Kyïv; Naukova dumka.

Transitions Online (2015). Crimea. The Anatomy of a Crisis. Prag; TOL.

UNHCR (2017): The situation of human rights in the temporarily occupied Autonomous Republic of Crimea and the city of Sevastopol (Ukraine), Kyiv; UNHCR.

Wilson, Andrew (2017): The Crimean Tatar Question. A Prism for Changing and Rival Versions of Eurasianism, Journal of Soviet and Post-Soviet Politics and Society, 3 (2), 1-46.

Zadorozhnii, Oleksandr (2016): Russian Doctrine of International Law after the Annexation of Crimea. Ohne Erscheinungsort: Amazon Digital Service. 